\title{
A review of technologies and applications for smart construction
}

Matej Štefanič BSc

Researcher, Faculty of Civil Engineering and Geodesy, University of Ljubljana, Slovenia
Vlado Stankovski PhD

Associate Professor, Faculty of Civil Engineering and Geodesy, University of Ljubljana, Slovenia (corresponding author: vlado.stankovski@fgg.uni-lj.si) (Orcid: 0000-0001-9547-787X)

The internet of things, artificial intelligence and cloud computing offer new opportunities for smart applications in many industries. Potential benefits include improvements of industrial processes, products and services, greater dependability and lower operational costs. This paper reports on an analysis of trends, opportunities and challenges for smart construction by conducting a review of recently published scientific articles. It provides an overview of emerging smart construction applications in areas such as construction monitoring, construction site management, safety at work, early disaster warning, and resources and assets management.

\section{Introduction}

The triumvirate of internet of things (IoT), artificial intelligence (AI) and cloud technologies offers new opportunities for the development of new applications in many industrial domains. It stimulates a new wave of highly dependable, available, reliable, safe, secure and performing smart applications. These technologies have already been used in parts or jointly to build applications that provide various benefits, such as improved logistics, safety and security in industrial settings. While smart applications have already been demonstrated in different industrial sectors, there is a general impression that the construction sector is lagging behind. An initial review of the literature showed a consistent lack of studies involving the joint use of IoT, AI and cloud technologies.

The aim of the work reported in this paper was therefore to study the trends, opportunities and challenges involved when developing and using smart applications in the construction sector. A specific goal of the work was to identify and present a comprehensive overview of existing application areas that demonstrate potential to benefit from these technologies. Although the review was focused on construction, its findings may also apply to the wider context of 'industry 4.0' (Wikipedia, 2018a), civil engineering, smart homes, cities, communities, transport and similar.

A representative body of papers on construction and information and communication technologies (ICT) published in the period from January 2012 to June 2018 in various construction- and information-technology-related journals was systematically reviewed. Section 2 discusses some key aspects of enabling ICT that are of great interest when building smart applications, while Section 3 presents some emerging areas for smart construction applications. Section 4 discusses the issues and challenges found and concludes the paper.

\section{Enabling ICT convergence}

In the first step of the review process, key enabling technologies and the level of their current convergence were identified. The literature review showed that IoT and the cloud technologies have already converged at a relatively high technology readiness level, while the level of integration of AI algorithms and methods in real-world applications is still somewhat lower. Nevertheless, this situation is expected to change rapidly due to the use of new open-source virtualisation, orchestration and elasticity mechanisms. A major enabler in this context is the use of software 'containers', such as Docker containers (Docker, 2018), as opposed to virtual machines. Containers have rapidly improving properties, such as their lightweight nature and operational speed, platform independence and efficient use of the underlying computing resources.

Many emerging smart applications involve the use of IoT devices, such as smartphones, cameras, sensors, actuators, cars and robots, and exert various degrees of operational autonomy. Building contextspecific smart environments is supported through the emergence of modular, cloud-native, open-source IoT platforms and interoperability standards, such as those of the Open Fog Consortium (2018) and the Cloud Native Computing Foundation (2018). By using methods and tools implementing such standards it has been possible to integrate physical devices and software quickly.

Various sophisticated, parallel and distributed AI algorithms (e.g. applications based on Tensorflow as an open-source software library for dataflow programming) are being implemented. The aim is to pack them in containers, deploy and orchestrate them across the whole computing continuum. The computing continuum starts from major data centres (i.e. cloud hubs) to smaller computing devices (e.g. routers and Raspberry Pi computers) close to the physical location of the smart environments (i.e. 'fog' devices), and ends at the actual 'edge', which is represented by wirelessly connected IoT devices. However, the technology readiness level of systems that apply AI methods on IoT-generated data flow, which is then processed in the cloud and fog, is very low.

The major challenge is that the triumvirate technologies IoT, $\mathrm{AI}$ and cloud must be designed to address various quality-ofservice requirements for data communication and processing. The IoT-generated data have properties such as huge volume, variety, velocity and veracity. To address these aspects, new cloudapplication-adaptation approaches, such as vertical and horizontal scaling, application migration and software-defined networking, aim at achieving smart application operation in time-critical situations.

Moreover, in many situations, multiple non-functional properties of the smart applications must be maintained, such as high performance, security, privacy, availability, reliability and dependability of the data, storage, computing and networking 
services. Emerging cloud, fog and edge technologies also aim at significant improvements of the utilisation of computing and networking resources, greater energy efficiency and use of renewable energy sources, lower operational costs and restricted operation to geographic areas, which is due to data protection regulations.

Nowadays, technology aims at enhanced transparency, security, automation of economic processes through the use of smart contracts and other benefits for smart applications. Due to all technological trends, 'devops' (Wikipedia, 2018b) as a new software engineering culture and practice has emerged. Devops aims at radical improvements of the software life cycle, thus making it increasingly possible to develop and deploy smart applications and environments rapidly.

\section{Emerging smart construction applications}

In the next step of the review process, identified advances in the mainstream ICT domain were related to a selection of papers addressing construction-related problems. Several potential smart application areas were identified, including construction monitoring, construction site management, safety at work, early warning against accidents and management of resources and assets.

A mind map summarising the identified application areas is shown in Figure 1. In the following sub-sections, representative works are presented.

\subsection{Construction monitoring}

Construction monitoring aims at preventing the occurrence of errors in the stability and durability of the buildings under construction. Only a few relevant applications have been identified, as follows.

An application has been developed to determine the exact position of the arm of a trencher (Lundeena et al., 2016). The identified position is used to prevent the tearing of wires, water pipes and gas pipes during operation. The developed algorithm calculates the difference between the existing mechanical, electrical and plumbing (MEP) systems and the intended operation area of the trencher.

One of the aims of the study was to develop a low-cost alternative to existing systems that determine the position of functional parts of excavators and trenchers. Instead of rotation sensors, which are usually embedded in construction machines or satellite-positioning components, this application uses a camera system and reference markers to determine the position of the individual moving elements, very similar to those seen in carcollision tests. With the current level of technology readiness, this application could be conveniently developed by using IoT and cloud technologies.

Another application (Liu et al., 2015) is used to facilitate automated measurements of the thickness of road layers. Its purpose is to prevent adverse effects on vehicles due to poorly maintained roads. This infrastructure-monitoring application is used to identify such road sections, properly mark them and use the information for road maintenance and signalisation. In the context of 'big data', an application like this can be integrated in road network information systems.

Construction vibrators are used widely in construction. They consist of an electric motor with eccentric rotation mass, packed into a hermetically sealed container, which causes vibrations while

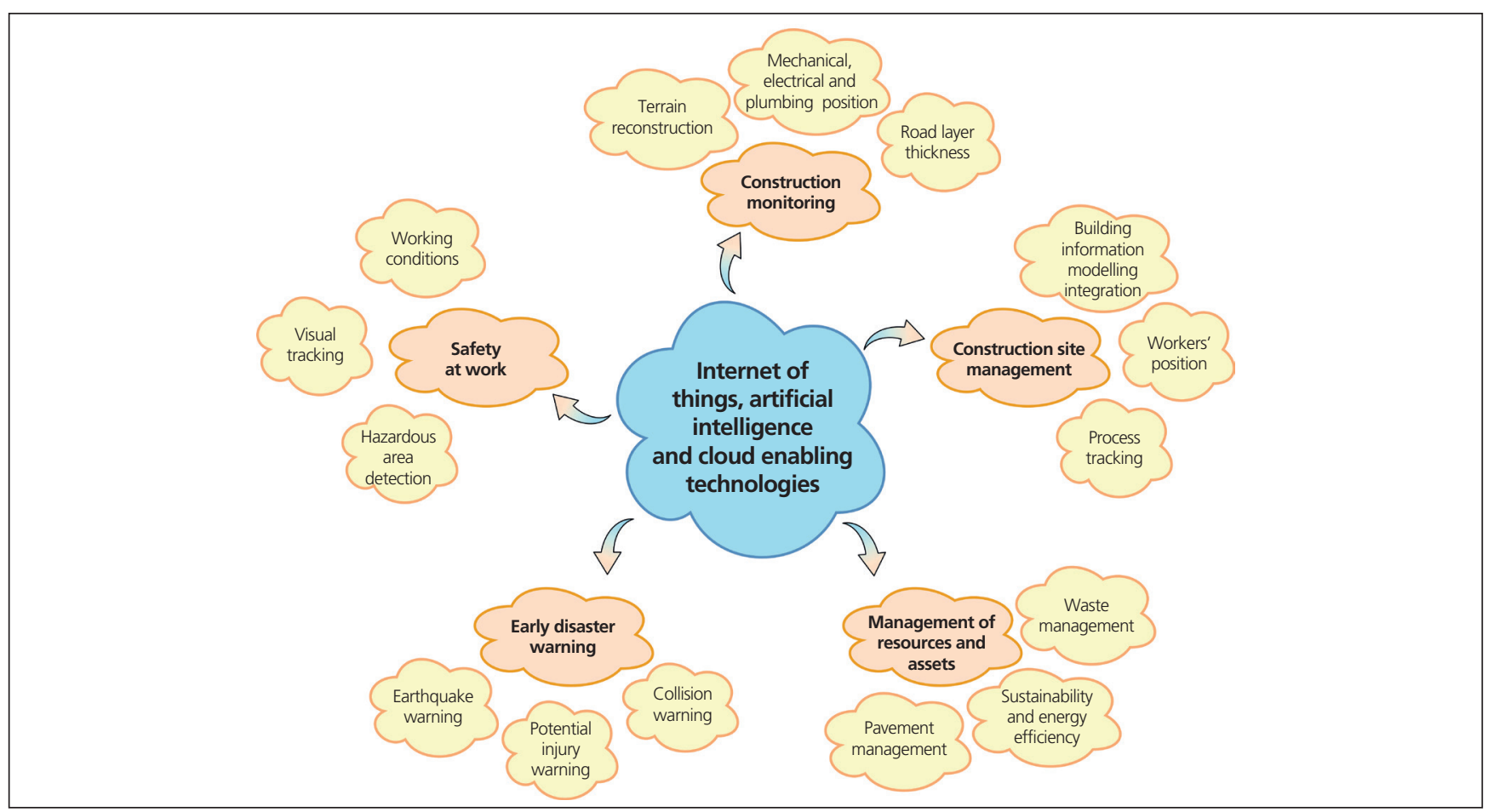

Figure 1. Converging internet of things, artificial intelligence, cloud, fog and edge computing technologies will provide the necessary dependability to time-critical smart construction applications 
spinning. They are used to remove packets of air trapped in poured concrete. An envisioned smart application (Gong et al., 2015) uses a real-time wireless-sensing-based internal vibrator tip tracking system that supports intelligent concrete consolidation operations. Using ultra-wideband radio waves, it determines the location of the construction vibrator, which prevents trapped air being missed. Real-time positioning is achieved through data integration from various sources, such as a combination of video streams and threedimensional (3D) data visualisation.

Video streams coming from stereo cameras have been used for 3D reconstruction of terrain (Sung and Kim, 2015). The resulting $3 \mathrm{D}$ images have then been used by construction workers to visualise current construction status. This represents a fast and robust 3D terrain-reconstruction system. The algorithms employed to analyse video streams start with the generation of multiscale descriptors (MSD), and continue with a plot consisting of triangles. The product of the application is a 3D real-time terrain reconstruction, which has the potential to become an integral part of many high-tech solutions in the construction industry.

\subsection{Construction progress tracking}

Numerous relevant studies have addressed construction site management problems. Generally, their goal is to achieve good insight into ongoing construction processes, while reducing the necessary time, subjectivity and errors due to manual work. A selection of such works is presented in the following.

An application automatically compares the state of a construction with its plan based on stereo photogrammetry (Braun et al., 2015). The collected data represent a dense point cloud. This requires the fusion of disparity maps created with semiglobal matching (SGM) with the target state provided by a fourdimensional (4D) building information model. An application like this requires the integration of various cameras with software tools, such as building information modelling (BIM) tools, which can be achieved seamlessly through the use of cloud technology.

An application has been developed for tracking, analysis and visualisation of as-built status of buildings under construction, and comparison against a 4D BIM model (Golparvar-Fard et al., 2012). Photographs taken daily from the construction site are used as input to the application. This application type is suitable for the use of AI methods such as Tensorflow.

Another application is used for progress tracking at construction sites (Turkan et al., 2011). 3D object models are built by using a 3D laser scanner. These are then combined with a 4D objectoriented progress-tracking system. The application demonstrates a high degree of accuracy for automated progress tracking that meets or exceeds typical manual performance. Additional integration with other data sources may contribute to even higher accuracy of the application.

Construction progress can be recorded in $4 \mathrm{D}$ computer-aided design (CAD) models, which may be updated timely and accurately during construction. This makes it possible to align progress with schedule (Kim et al., 2013).

Video analysis can be used to determine workers' positions from video clips (Park and Brilakis, 2012). The application does not require any additional equipment for the tracking. The analysis of the video is sufficient since specifically implemented algorithms recognise movements, shape and colour. The application can be used for, but is not limited to, measuring productivity of workers, analysing the sequence of activities and improving site safety. Dependability is obviously an important property of applications such as this one, which must be obtained when realising the same software service by way of the cloud. This, however, is a nontrivial problem in the cloud computing domain.

\subsection{Safety at work}

Ensuring a safe and healthy environment for workers is the main collective concern of construction companies (Walker, 2014). It involves both humanitarian and economic considerations. In the literature, various applications involving safety and health aspects were found. Some of these are presented in the following.

Construction may take place in hot weather, where a combination of high temperature and high moisture can pose a risk to the workers' health. An application has been built to warn about extreme work conditions (Yi et al., 2015). It uses sensor data including moisture, temperature, the workers' on-site location and their heart rates. A decision algorithm based on a neural network also takes into account the exposure time and basic health data of individual workers.

Automatic detection of hazardous areas on construction sites may prevent many injuries. A new application has been built (Teizer and Cheng, 2015) to compare optimal workers' paths across construction sites (the shortest possible footprints obtained from a BIM model) with actual tracking of workers' paths, with a real-time positioning system. Differences in these two paths reveal obstacles and potentially dangerous areas on the site. Consequently, the site security plan is continuously updated and does not solely depend on the security engineer.

In addition to perceiving the location of workers, warning about the proximity of hazardous areas on the construction site may also improve construction site safety. An application has been developed to generate spatial-time data on construction machines and material on construction sites (Kim et al., 2016). After analysis, individual workers are issued warnings about the proximity of dangerous areas, while security inspectors and construction site managers get data analysis and automated reports.

Implementation of accelerometers to detect near-falls of workers is a solution focused on installers of steel structures and welders, who often work at great height (Yang et al., 2016). Each worker carries a device containing an accelerometer, a gyroscope and a magnetometer - a wireless inertial measurement unit (WIMU). The system helps detect dangerous areas at the construction site by detecting sudden acceleration.

One way to improve security at construction sites is to increase collective knowledge, which can be achieved by establishing a social network dedicated to the exchange of data on work-related accidents (Le et al., 2014). The application archives data about falls from height and classifies and analyses the data statistically. It can quickly and accurately identify weak points in protecting the life and health of workers at construction sites. The integration of smart construction environments with the world wide web is therefore a necessity, which contributes to greater safety.

Using a similar approach to the smart construction vibrator application, a new application has been developed to determine the position of construction elements through ultra-wideband radio waves (Hwang, 2011). In this approach, sensors are positioned on the functional parts of the cranes, and the information is used to prevent collisions. 


\subsection{Early disaster warning}

In the course of the research, cases were found of various early-warning systems. These have wider applicability, as can be observed from the following example applications. Although not always directly related to construction, such systems could be greatly enhanced by combining IoT, AI and cloud technologies.

Preventing collisions reduces danger of injuries of workers, machinery and part-built structures. To reduce the rate of collision accidents between mobile cranes and immobile obstacles in construction sites during lift operations, a real-time automated anti-collision system has been developed (Ren and $\mathrm{Wu}, 2014)$. The system is used to warn crane operators about potential collisions and automatically implements collision-avoidance strategies. Most mobile cranes already have sensors for arm extension, rotation and cord length. The information from such sensors presents an input to a decision-making module. Before starting the operation, the existing system determines the area where trucks can safely operate. The core of the existing system is a decision module that, on the basis of input spatial data, issues warnings when approaching sensitive areas.

An earthquake-detection system conveniently takes advantage of accelerometers that are built into most smart phones (Zambrano et al., 2014). Thus, in a certain seismic area, a network of low-cost wireless sensors is created, which in the event of an earthquake performs specific numerical analyses. The result is up to $12 \mathrm{~s}$ faster determination of the epicentre and issuing of a warning, and fewer false positives. In another application, the seismograph network (Böse et al., 2014) detects longitudinal waves and calculates the epicentre of an earthquake. It takes advantage of the speed difference and informs the user of the arrival time of transversal waves.

\subsection{Management of resources and assets}

Management of resources and assets is an important area of construction. Several applications have been developed to address various related problems and a selection of these is presented in the following.

In areas critical to groundwater contamination, periodic reviews of the level and quality of groundwater are often carried out. An application uses as input the height of groundwater in individual wells and the results of chemical analysis of water samples (Jones et al., 2014). It then performs statistical analyses and graphically displays spatial time results. The application is used as a tool to control the environment of oil refineries.

A new web site focuses on construction site waste management (Li and Zhang, 2013). It is used to assess the type and quantity of construction waste. The input data are a timetable and a breakdown of the work structure. The application calculates the quantity and types of wastes, and the time when they will have to be removed from a construction site.

A system for covering pavement management has been developed (Radopoulou and Brilakis, 2015). It relies on comprehensive up-to-date road-condition data. The level of wear of the upper layer is determined by periodical checks, which are generally rare due to the distances involved. The described system takes advantage of cars' inbuilt parking cameras, which are used to scan the road surface. The system then automatically detects and assesses the damage on the carriageway.

An application has been developed (König et al., 2013) by using the latest linked data technologies of the 'semantic web' (Wikipedia, 2018c). It is used in the design process of the building envelope.
It evaluates the energy efficiency, carbon dioxide emissions of the production process and other high-level quality parameters of various building materials, composites and final products.

\section{Discussion and conclusions}

The development of smart applications in the identified problem areas requires the integration of complex physical and software resources, starting from sensors and cameras, through to software for processing time-series and video streams, AI methods for features extraction, data fusion, and visualisation software. Moreover, specific tools are required for the management of non-functional properties of applications, including their dependability, security and privacy.

Many smart construction applications are time-critical in nature and must be designed having in mind this requirement (see Figure 2). Accordingly, each new smart construction site involving potentially hundreds of smart applications will have to be planned in advance. Designing time-critical smart applications is particularly challenging due to the dynamic nature of construction - every project has its unique goals, size, equipment, workers, data and problems to be solved.

Moreover, some end-user requirements may be conflicting in nature - for example, fast application performance may conflict with the requirement for low operational costs, or data-fusion requirements may conflict with data-privacy requirements.

Unfortunately, big-data analytic platforms that are commonly in use by the finance and pharmaceutical industries cannot be applied directly to the domain of construction. It would require an additional cycle of technology development and integration to enable a big-data pipeline starting from dynamically changing smart construction environments.

One way to address the range of problems is through the use of modern software workbenches for time-critical cloud applications. With them and by following the devops culture, it may soon become possible seamlessly to integrate IoT devices and containerbased functionality (such as AI methods) and deploy the smart applications across cloud and fog providers in a way that addresses critical requirements.

The emerging vision is that data coming from field sensors, including video streams, will first be processed and fused in fog devices that are geographically close to an actual construction site. This approach

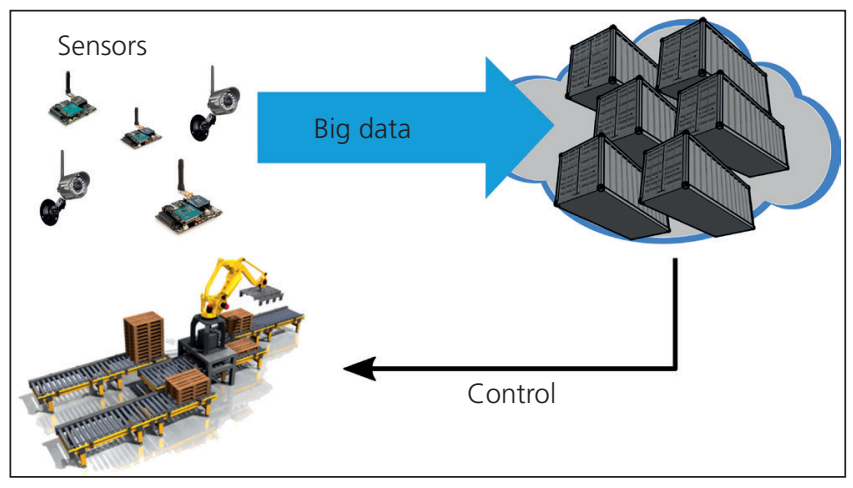

Figure 2. Construction problem areas and sets of technology solutions 
would address important dependability and safety requirements. By using the fog computing approach, network-based quality-of-service metrics - such as bandwidth, latency, jitter and packet loss - will be improved significantly (Kochovski and Stankovski, 2018).

The use of container-based virtualisation will also make it possible to deploy and operate various AI algorithms and software closer to the data streams, thus reducing the necessity for large traffic across the network and addressing data-privacy and security requirements, which are necessary for the operation of smart construction environments.

The review of scientific papers revealed great application potential for the construction area on the one hand, and a very low level of latest technology usage on the other. There are major benefits which are still expected from the integration of IoT, AI and cloud technologies, including a high level of dependability, performance, availability, reliability, automation, data security and privacy, addressing time-critical aspects, improved energy efficiency and reduced operational costs. These are yet to be demonstrated in application scenarios, such as those presented in this paper.

Future work by the authors will concentrate on the design and implementation of a single smart construction site, addressing several identified problem areas.

\section{Acknowledgements}

The research and development reported in this paper have received funding from the European Union's Horizon 2020 Research and Innovation Programme under grant agreements no. 643963 (SWITCH project: Software Workbench for Interactive, Time Critical and Highly self-adaptive cloud applications) and no. 815141 (DECENTER: Decentralised technologies for orchestrated cloud-toedge intelligence). Funding was also received from the Slovenian Research Agency under grant agreement no. BI-RU/1618-043 (Internet of Things and cloud computing as support for the development of new smart approaches in the construction sector).

\section{References}

Braun A, Tuttas S, Borrmann A and Stilla U (2015) A concept for automated construction progress monitoring using BIM-based geometric constraints and photogrammetric point clouds. Information Technology in Construction 20: 68-79.

Böse M, Allen R, Brown $\mathrm{H}$ et al. (2014) CISN ShakeAlert: an earthquake early warning demonstration system for California. In Early Warning for Geological Disasters: Scientific Methods and Current Practice (Wenzel F and Zschau J (eds)). Springer, Berlin, Germany, pp. 49-69.

Cloud Native Computing Foundation (2018) https://www.cncf.io/ (accessed 13/11/2018).

Docker (2018) https://www.docker.com/ (accessed 13/11/2018).

Golparvar-Fard M, Peña-Mora F and Savarese S (2012) Automated progress monitoring using unordered daily construction photographs and IFC-based building information models. Journal of Computing in Civil Engineering 29(1): 04014025.

Gong J, Yu Y, Krishnamoorthy R and Roda A (2015) Real-time tracking of concrete vibration effort for intelligent concrete consolidation. Automation in Construction 54: 12-24.

Hwang S (2011) Ultra-wide band technology experiments for real-time prevention of tower crane collisions. Automation in Construction 22 545-553.
Jones WR, Spence MJ, Bowman AW, Evers L and Molinari DA (2014) A software tool for the spatiotemporal analysis and reporting of groundwater monitoring data. Environmental Modelling \& Software 55: 242-249.

Kim C, Kim B and Kim H (2013) 4D CAD model updating using image processing-based construction progress monitoring. Automation in Construction 35: 44-52.

Kim H, Lee HS, Park M, Chung B and Hwang S (2016) Automated hazardous area identification using laborers' actual and optimal routes. Automation in Construction 65: 21-32.

Kochovski P and Stankovski V (2018) Supporting smart construction with dependable edge computing infrastructures and applications. Automation in Construction 85: 182-192.

König M, Dirnbek J and Stankovski V (2013) Architecture of an open knowledge base for sustainable buildings based on linked data technologies. Automation in Construction 35: 542-550.

Le QT, Lee DY and Park CS (2014) A social network system for sharing construction safety and health knowledge. Automation in Construction 46: 30-37.

Li Y and Zhang X (2013) Web-based construction waste estimation system for building construction projects. Automation in Construction $\mathbf{3 5}$ $142-156$

Liu D, Wu Y, Li S and Sun Y (2015) A real-time monitoring system for liftthickness control in highway construction. Automation in Construction 63: $27-36$

Lundeena KM, Dong S, Fredricks N et al. (2016) Optical marker-based end effector pose estimation for articulated excavators. Automation in Construction 65: 51-64.

Open Fog Consortium (2018) https://www.openfogconsortium.org/ (accessed 13/11/2018)

Park MW and Brilakis I (2012) Construction worker detection in video frames for initializing vision tracker. Automation in Construction 28: 15-25.

Radopoulou SC and Brilakis I (2015) Patch detection for pavement assessment. Automation in Construction 53: 95-104.

Ren W and Wu Z (2014) Real-time anticollision system for mobile cranes during lift operations. Journal of Computing in Civil Engineering 29(6) 04014100

Sung C and Kim PY (2015) 3D terrain reconstruction of construction sites using a stereo camera. Automation in Construction 64: 65-77.

Teizer J and Cheng T (2015) Proximity hazard indicator for workers-on-foot near miss interactions with construction equipment and geo-referenced hazard areas. Automation in Construction 60: 58-73.

Turkan Y, Bosche F, Haas CT and Haas R (2011) Automated progress tracking using 4D schedule and 3D sensing technologies. Automation in Construction 22: 414-421.

Walker $\mathrm{G}$ (2014). New reports highlight the critical role of health and safety in professional engineering. Proceedings of the Institution of Civil Engineers - Civil Engineering 167(1):12, https://doi.org/10.1680/ cien.2014.167.1.12.

Wikipedia (2018a) https://en.wikipedia.org/wiki/Industry_4.0 (accessed 13/11/2018)

Wikipedia (2018b) https://en.wikipedia.org/wiki/DevOps (accessed 13/11/2018)

Wikipedia (2018c) https://en.wikipedia.org/wiki/Semantic_Web (accessed 13/11/2018)

Yang K, Ahn CR, Vuran MC and Aria SS (2016) Semi-supervised near-miss fall detection for ironworkers with a wearable inertial measurement unit. Automation in Construction 68: 194-202.

Yi W, Chana APC, Wang X and Wang J (2015) Development of an earlywarning system for site work in hot and humid environments: A case study. Automation in Construction 62: 101-113.

Zambrano A, Perez I, Palau C and Esteve M (2014) Quake detection system using smartphone-based wireless sensor network for early warning. In 2014 IEEE International Conference on Pervasive Computing and Communication Workshops (PERCOM WORKSHOPS). Institute of Electrical and Electronics Engineers, New York, NY, USA, pp. 297-302. 